\section{The relationship between human leukocyte antigen-cw6 allele and psoriasis vulgaris}

\author{
Sri Lestari KS, ${ }^{1}$ Eryati Darwin, ${ }^{2}$ \\ Tjut Nurul Alam Jacoeb, ${ }^{3}$ \\ Djong Hon Tjong ${ }^{4}$
}

${ }^{1}$ Department of Dermatology and Venereology, Medical Faculty of Andalas University, Padang; ${ }^{2}$ Department of Histology, Medical Faculty of Andalas University, Padang; ${ }^{3}$ Department of Dermatology and Venereology, Medical Faculty of Indonesia University, Jakarta; ${ }^{4}$ Mathematics and Natural Science Faculty of Andalas University, Padang, Indonesia

\begin{abstract}
Psoriasis vulgaris is chronic skin disease that is linked to genetics and immune system. The most important predisposing genetic factor is human leukocyte antigen (HLA). This study was performed to determine the relationship between HLA-Cw6 allele and psoriasis vulgaris and the changes of nucleotide base squences, using observation method with a cross sectional comparative study. Samples were selected using consecutive sampling of 30 patients with psoriasis vulgaris attending the Dermatology and STD polyclinic at DR. M. Djamil Hospital. 30 healthy volunteers were selected as controls. The subjects' medical history was recorded followed by a dermatological examination, collection of samples, DNA isolation, then primers were designed for HLA-Cw6 allele, genes were sequencing and finally analyzed using PCR-SSP. The results were $20 \%$ of patients with psoriasis vulgaris carried HLA-Cw6 allele, while it was absent in the control group. This difference is statistically significant at the $5 \%$ level $(\mathrm{p}=0.024)$. We found the changes of nucleotide base formations of HLA-Cw6. In conclusion, based on these observations, presence of the HLA-Cw6 allele is an important genetic risk factor for developing psoriasis vulgaris.
\end{abstract}

\section{Introduction}

Psoriasis vulgaris is a chronic skin disease that interferes with daily life resulting in high levels of morbidity. ${ }^{1}$ It affects a patient's appearance ${ }^{2}$, reducing their quality of life $\mathrm{e}^{1,2}$, and impacting both the social and health systems. ${ }^{1}$ Globally the incidence is 2$3 \%$ of the population. ${ }^{3,4}$ Amongst patients at the Dermatology and STD polyclinic at the DR. M. Djamil Hospital in Padang, the prevalence of patients diagnosed with psoriasis is increasing; $2.8 \%$ in 2014 and 3.6\% in 2016. Psoriasis is an autoimmune disease that is mediated by $\mathrm{T}$ cells, characterized by hyperproliferation of keratinocytes and is related to HLA (human leukocyte antigen) particularly the HLA-Cw6 allele. ${ }^{5}$ Psoriasis is the result of an interaction between this hyperproliferation of keratinocytes and the infiltration and activation of immune cells. ${ }^{4}$

The human leucocyte antigen (HLA) system is a gene locus that encodes a cell wall protein responsible for the regulation of the human immune system. Human leucocyte antigen-Cw6 is a class one variant of MHC (major histocompatibiy complex) related to the early onset of psoriasis vulgaris and primarily responds with a $\mathrm{T}$ cell expression of $\mathrm{CD}-8+$. Type I psoriasis occurs in patients before 40 years of age, often reoccurs, runs in families, has a more severe clinical presentation and is primarily related to HLA-Cw6. Type II psoriasis has a later onset, does not occur in family histories and clinical symptoms are less severe. It is related to HLA-Cw2 and -B27, with only a weak correlation with HLA-Cw6. ${ }^{6}$

HLA-Cw6 incidence varies with racial background and geography and also this chronic recidive disease is hard to treat. Hence, this study focused on the role of HLA-Cw6 in psoriasis vulgaris patients and the changes of nucleotide base squences.

\section{Materials and Methods}

Consecutive random sampling was used to select from the subjects fulfilling the criteria. A physical examination was conducted to determine the severity of the psoriasis (PASI score). A 5cc blood sample was taken from the cubital vein of each subject. This was used to check for the presence of HLA-Cw6 using a sequencespecific primer. DNA isolation was conducted using a Vivantis GF-1 Blood/ Cultured Cell DNA Geneaid Cat.No GB100 kit. Synthesis of the primer was done by Integrated DNA Technologies (IDT) Singapore. The reagent for PCR was KAPATaq Extra Hot Start (Cat. No. KK3606).

\section{Results}

Table 1 shows the characteristics of subjects in the two samples. Subject age was $51.0 \pm 15.1$ with a mode of 46 to 56 years $(23.3 \%)$. All subjects were 12 years old or above. Males slightly outnumbered females $(53.3 \%$ compared to $46.7 \%)$
Correspondence: Sri Lestari KS, Department of Dermatology and Venereology, Medical Faculty of Andalas University, Air Sirah Street, no. 14, Jati Baru, Padang 25128, West Sumatera, Indonesia.

Phone: 08126603159

E-mail: srilestari07@yahoo.com

Key words: Psoriasis vulgaris, HLA-Cw6 allele.

Contributions: The authors contributed equally.

Conflict of interest: the authors declare no potential conflict of interest.

Received for publication: 1 February 2019. Accepted for publication: 13 February 2019.

This work is licensed under a Creative Commons Attribution-NonCommercial 4.0 International License (CC BY-NC 4.0).

(C) Copyright S.L. Ks et al., 2019

Licensee PAGEPress, Italy

Dermatology Reports 2019; 11(s1):8054

doi:10.4081/dr:2019.8054

amongst psoriasis sufferers. $43.0 \%$ of psoriasis patients and $43.3 \%$ of control subjects had junior high school education and half of each group had a tertiary education. Table 2 shows $76.7 \%$ of patients had mild psoriasis, $16.7 \%$ moderate psoriasis and $6.6 \%$ had severe psoriasis, $76.7 \%$ first suffered the disease at or before the age of 40 while only $23.3 \%$ first experienced it after this age. $50 \%$ had suffered from the disease from between 5 and 15 years, $46.7 \%$ for five years or less and only $3.3 \%$ had suffered from psoriasis for more than 15 years. Bioinformatic study of the HLA-Cw6 was conducted using primer designed by Bunce \& Welsh (1994). The appropriateness of the primer was verified by alignment with the Homo Sapiens HLA-C gene sequence (NCBI Accession NG_029422.2) using the Geneious (version 7.0.6) software. Tables 3 and 4 show the primer for HLA-Cw6. PCR products are 336 bp respectively (adapted from Bunce \& Welsh, 1994) (Figures 1-3).

\section{Discussion}

The literature suggests that the onset of psoriasis vulgaris can occur at any age from infancy until the seventies. There are two peaks for onset age, between 20-30 and 50$60 .^{7,8}$ Henseler and Christopher $(1985)^{9}$ divided psoriasis into two types. Type 1 usually has an onset before the age of 40 and is coincident with the HLA-Cw6 allele in $85 \%$ of the cases so runs in families. Type II has a later onset and is unrelated to HLA. 
Bolognia et al. (2004) found that $75 \%$ of all psoriasis vulgaris cases in their study were early onset. ${ }^{8}$ Sommer et al. (2006) also found that psoriasis type I $(60.2 \%)$ was more common that type II $(36.9 \%){ }^{10}$ Similarly, over three quarters $(76.7 \%)$ of the patients in our study first contracted psoriasis vulgaris before they were 40 years old.

Psoriasis vulgaris afflicts males and females equally. Most psoriasis patients in this study were aged between 46 and 56 and the number of males and females was almost the same $(53.3 \%$ and $46.7 \%)$. A dis- proportionate number of patients had higher levels of education and the majority had mild cases of the disease. This maybe because more educated and social aware sufferers feel embarrassed about this disease so seek early treatment. More than half the patients in our study had suffered from the disease for more than 5 years. This is in line with the chronic recidive nature of psoriasis vulgaris reported in the literature.

The presence of HLA-Cw6 in 20\% of the psoriasis vulgaris patients and not in the control group compares with the findings of
Amr et al., (2010) that amongst Egyptians. HLA-Cw6 was more common $(86 \%)$ in psoriasis patients than in the control group (40\%). Gudjonsson et al., (2001) reported that of 369 Icelandic psoriasis vulgaris patients $(66.9 \%)$ were HLA-Cw6 positive and 122 were HLA-Cw6 negative. Atasoy et al., (2006) found that 48 of $72(66.9 \%)$ Turkish patients were HLA-Cw6 positive but only 9 of $104(8.7 \%)$ of control subjects. ${ }^{11}$

High resolution PCR studies have shown that HLA-Cw*0602 is the primary
Table 1. Age, gender, and level of education of subjects with psoriasis vulgaris and control.

\begin{tabular}{|c|c|c|c|c|}
\hline \multirow[t]{2}{*}{ Characteristics } & \multicolumn{2}{|c|}{ Psoriasis Vulgaris } & \multicolumn{2}{|c|}{ Non-Psoriasis Vulgaris } \\
\hline & n & $\%$ & n & $\%$ \\
\hline \multicolumn{5}{|l|}{ Age } \\
\hline $12-<17$ & 1 & 3.3 & 0 & 0 \\
\hline$>17-<26$ & 5 & 16.7 & 6 & 20 \\
\hline$>26-<36$ & 5 & 16.7 & 5 & 16.7 \\
\hline$>36-<46$ & 6 & 20 & 6 & 20 \\
\hline$>46-<56$ & 7 & 23.3 & 7 & 23.3 \\
\hline$>56-<65$ & 6 & 20 & 6 & 20 \\
\hline$>65$ & 0 & 0 & 0 & 0 \\
\hline Sum & 30 & 100 & 30 & 100 \\
\hline \multicolumn{5}{|l|}{ Gender } \\
\hline Male & 16 & 53.3 & 16 & 53.3 \\
\hline Female & 14 & 46.7 & 14 & 46.7 \\
\hline Sum & 30 & 100 & 30 & 100 \\
\hline \multicolumn{5}{|l|}{ Level of Education } \\
\hline Elementary School & 4 & 13.3 & 0 & 0 \\
\hline Junior High School & 5 & 16.7 & 0 & 0 \\
\hline Senior High School & 13 & 43.3 & 15 & 50 \\
\hline Tertiary Education & 8 & 26.6 & 15 & 50 \\
\hline Sum & 30 & 100 & 30 & 100 \\
\hline
\end{tabular}

Table 2. Degree of severity, onset and duration of psoriasis vulgaris in each patient.

\begin{tabular}{lcc} 
Characteristic & Psoriasis Vulgaris \\
Severity & & \\
$\quad$ Mild (PASI <8) & 23 & 76.7 \\
Moderate (PASI >8-<12) & 5 & 16.7 \\
Severe (PASI >12) & 2 & 6.6 \\
Sum & 30 & 100 \\
Onset of disease & & \\
Before age 40 & 23 & 76.7 \\
After age 40 & 7 & 23.3 \\
Sum & 30 & 100 \\
\hline Duration & & \\
$\quad<5 y e a r s$ & 14 & 46.7 \\
between $>5$ and $<15 y e a r s ~$ & 15 & 50 \\
$\quad>15 y e a r s$ & 1 & 3.3 \\
Sum & 30 & 100 \\
\hline
\end{tabular}

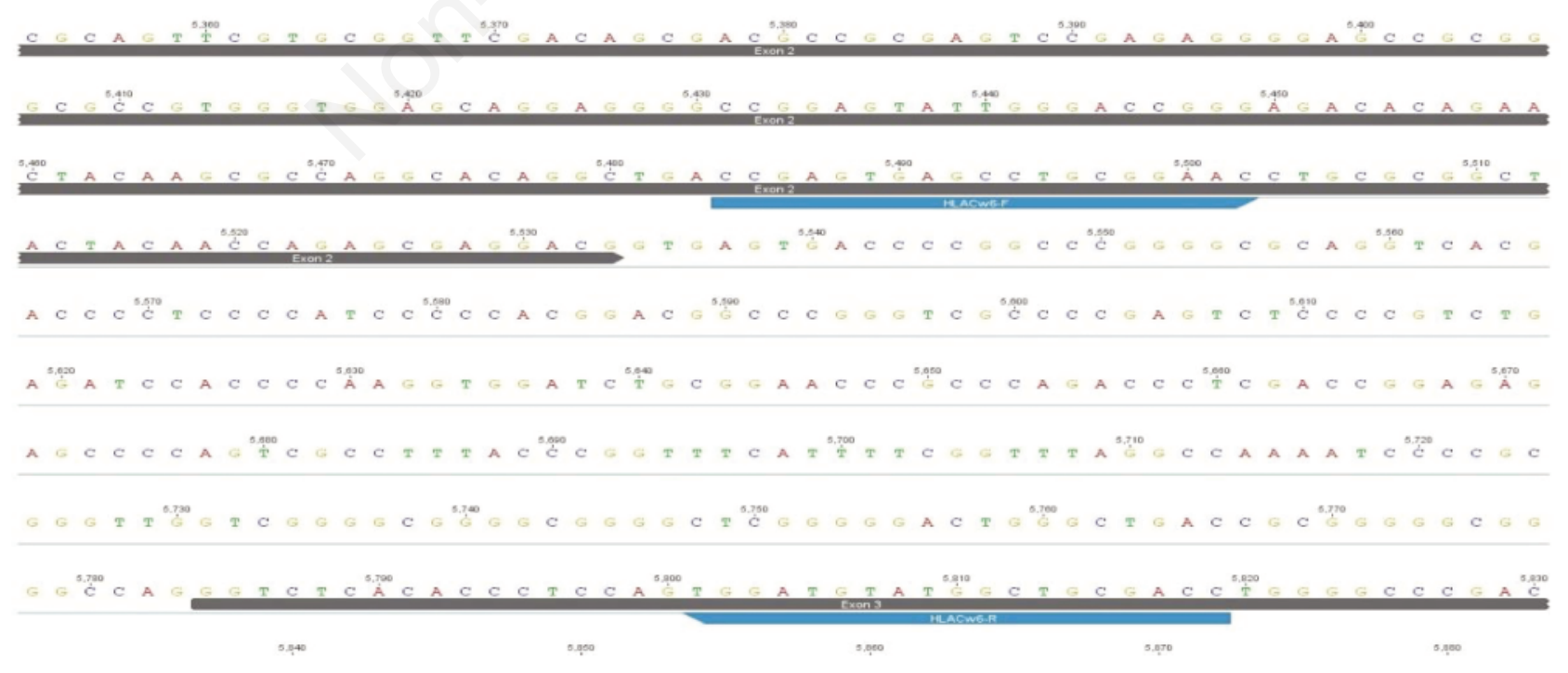

Figure 1. Result of HLA-Cw6 allele primer design. HLA-C (NG_029422.2) gene sequence and HLA-Cw6 attachment position, forward and reverse. HLA-Cw6-F primer attaches from the 5,484th to the 5,502th nucleotide bases. The HLACw6-R primer attaches from the 5,801 th to the 5,5819th nucleotide bases. Amplification used this primer pair to produce a 336 bp PCR product. 
Table 3. Results from primer for HLA-Cw6 allele

\begin{tabular}{llll}
\hline HILA Allele & Primer Name & Nucleotide Sequence Size of PCR product \\
Cw*0601,0602, CL10v & HLA-Cw6-F & 5'-CCGAGTGAACCTGCGGAAA-3' & \multirow{2}{*}{$336 \mathrm{bp}$} \\
& HLA-Cw6-R & 5'-GGTCGCAGCCATACATCCA-3' & \\
\hline
\end{tabular}

Table 4. Relationship of presence of HLA-Cw6 in patients with psoriasis vulgarisand control subjects. HLA-Cw6 was present in 6 of the 30 patients with psoriasis $(20 \%)$ and absent in the control group. There is a statistically significant relationship between the presence of HLA-Cw6 with psoriasis vulgaris $(\mathrm{p}<0.05)$ so it can be concluded that HLACw6 plays a role and resulting incidence of psoriasis vulgaris.

\begin{tabular}{|c|c|c|c|c|c|c|c|}
\hline \multirow[t]{2}{*}{ Subject } & \multicolumn{6}{|c|}{ HLA-Cw6 } & \multirow[t]{2}{*}{$P$} \\
\hline & $\mathbf{N}$ & $\%$ & N & $\%$ & n & $\%$ & \\
\hline Patient & 6 & 20 & 24 & 80 & 30 & 100 & 0.024 \\
\hline Control & 0 & 0 & 30 & 100 & 30 & 100 & 0.024 \\
\hline Sum & 6 & 10 & 54 & 90 & 60 & 100 & 0.024 \\
\hline
\end{tabular}

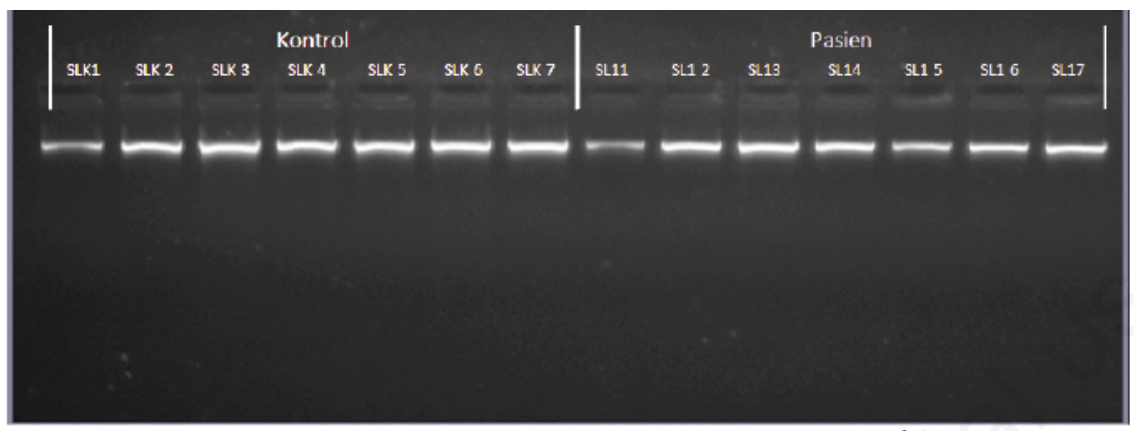

Figure 2. Result of DNA isolation from all subjects using electrophoregram. The genomic DNA electrophoregram of $5 \mu \mathrm{L}$ samples after electrophoresis in $1.5 \%$ agarose gel stained with DNA GelRed. The DNA band is visible on each sample. This shows the genomic DNA has been successfully isolated.
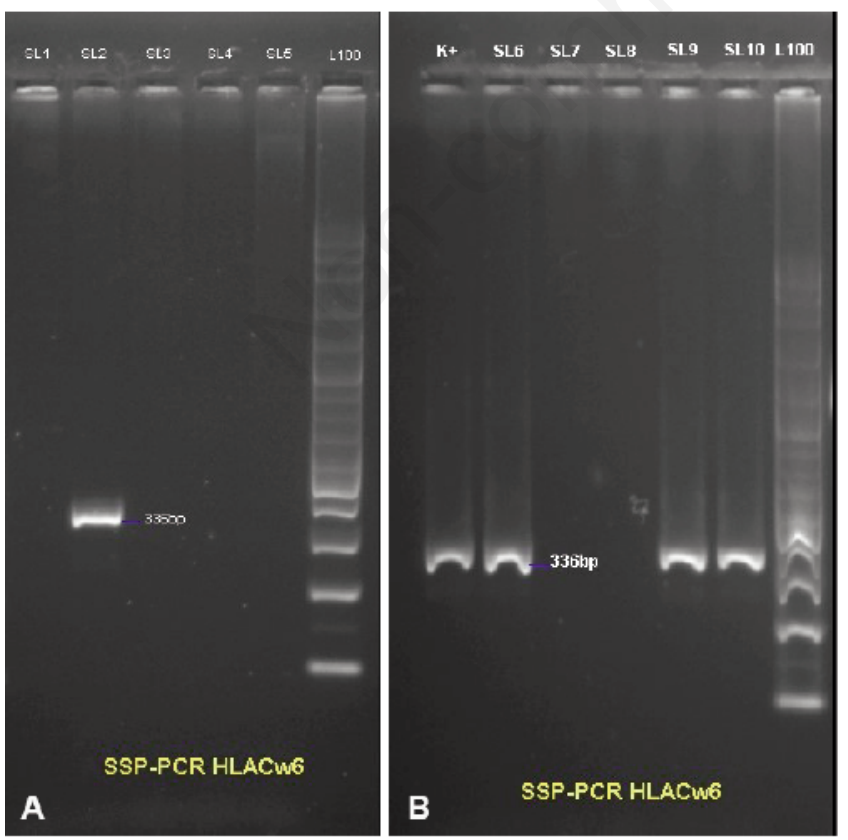

Figure 3. HLA-Cw6 PCR electrophoregram. Isolated genomic DNA forms the template in the reaction. DNA band is visible at \pm 336 bp compared to the DNA size marker or the 100 bp (L100) DNA Ladder, then the sample can be said to be positive for HLA-Cw6, for example samples coded SL2, SL6, SL9, and SL10. Conversely, an absence of a band in that position corresponds to an absence of the allele as in SL1, SL3, SL4, SL5, SL7, and SL8. allele for suseptability to psoriasis vulgaris in West India. ${ }^{5}$ Human leucocyte antigenCw6 has the ability to produce peptides and trigger a CD8+ T cell response. ${ }^{12}$ HLA-Cw6 is a highly significant marker for the predicting risk of developing psoriasis vulgaris. ${ }^{13}$ A study amongst the Chinese population reported that a HLA-Cw6 positive individual has an $8.33 \mathrm{x}$ greater risk of developing psoriasis compared to individuals who are HLA-Cw6 negative. ${ }^{14}$ Chandra et al., found a significant relationship between HLA-Cw6 and psoriasis vulgaris. From 705 cases of psoriasis, $61.74 \%$ had type I (early onset). 53\% of these and 34\% of those with type II were HLA-Cw6 positive but only $14.85 \%$ of the control group. This last result differs from previous work that showed a significant relationship between HLA-Cw6 and both types of psoriasis vulgaris compared to the control even though the risk associated with HLA-Cw6 for Type I is twice that of Type II. ${ }^{15}$

\section{Conclusions}

In this research HLA-Cw6 was only found in psoriasis vulgaris patients, not in the control group. We also found some changes of nucleotide base sequences. More research is needed to investigate the other genetic and immunological factors that play a role in clinical psoriasis vulgaris.

\section{References}

1. Wu D, Wu Y, Liu JL, Wang B, Zhang XD. Association between HLA$\mathrm{CW}^{*} 0602$ polymorphism and psoriasis risk: A meta-analysis. Genetic and Molecular Reearch. 2011;10(4): 31093120 .

2. Eskicirak B, Zemheri E, Cerkezoglu A. The threatment of psoriasis vulagris: $1 \%$ topical methotrexate gel. International Journal of Dermatology. 2006;45:965-969.

3. Gaspari AA. Innate and adaptive immunity and the pathophysiology of psoriasis. J Am Acad Dermatol. 2006;54:S67-80.

4. Harden JL, Krueger JG, Bowcock AM. The immunogenetic of psoriasis: A comprehensive review. Journal of Autoimmunity. 2015:1-8.

5. Shankarkumar U. HLA C allele associations in Mumbai psoriasis patients. Int $\mathrm{J}$ Hum Genet. 2012;12(1):41-44.

6. Bahcetepe N, Kutlubay Z, Yilmaz E, Tuzun Y, Eren B. The role of HLA antigens in the aetiology of psoriasis. Med Glas (Zenica). 2013;10(2):339- 
342.

7. Bowcock AM, Cookson WOCM. The genetic of psoriasis, psoriatic arthritis and atopic dermatitis. Human Molecular Genetic. 2004;13 Review Issue I: R43-R55.

8. Bolognia JL, Jorizzo JL, Rapini RP. 2004. Psoriasis. In: Dermatology. Vol 1. Horn TD, Mancini AJ, Mascaro JM, Salasche SJ, Saurat JH, Stingl G eds. Edinburg, Mosby:125-149.

9. Henseler T, Christophers E. Psoriasis of early and late onset, characterization of two types of psoriasis vulgaris. J Am Acad Dermatol. 1985;13:578-582.

10. Sommer DM, Jenisch S, Suchan M, Christophers E, Weichental M. Increased prevalence of the metabolic syndrome in patients with moderate to severe psoriasis. Arch Dermatol Res. 2006;298(7):321-328.

11. Amr KS, Weshahy AH, El Hadidi HH, El Hamid MFA, Oteafy MR, Aly DG. A pilot study of HLA-Cw6 association with Egyptian psoriatic patients. Research Journal of Medicine and Medical Sciences. 2010;5(1):25-29.

12. Hundhausen $C$, Bertoni A, Mak RK, Botti E, Meglio PD, Clop A, et al. Allele-specific cytokine, responses at the HLA-C locus, implications for psoriasis. J Invest Dermatol. 2012;132(301):635-641.

13. Fan X, Yang S, Huang W, Wang ZM, Sun LD, Liang YH, et al. Fine mapping of the psoriasis susceptibility locus
PSORS1 supports HLA-C as the susceptibility gene in the Han Chinese population. PLOS Genetics. 2008;4(3):1-10.

14. Jin Y, Zhang F, Yang S, Kong Y, Xiao F, Hou Y, et al. Combined effect of HLACw6, body mass index and waist-hip ratio onpsoriasis vulgaris in Chinese Han population. Journal of Dermatologycal Science. 2008;52(2):123-129.

15. Chandra A, Lahiri A, Senapati S, Basu B, Ghosh S, Mukhopadhyay I, et al. Increased risk of psoriasis due to combined effect of HLA-Cw6 and LCE3 risk alleles in Indian population. 2016:1-8. 